\title{
The sphere
}

From pure logic and imagination, we construct a sphere upon the real world. From the top, a bright spot shines through the surface, and shine on the real world the sphere's image.

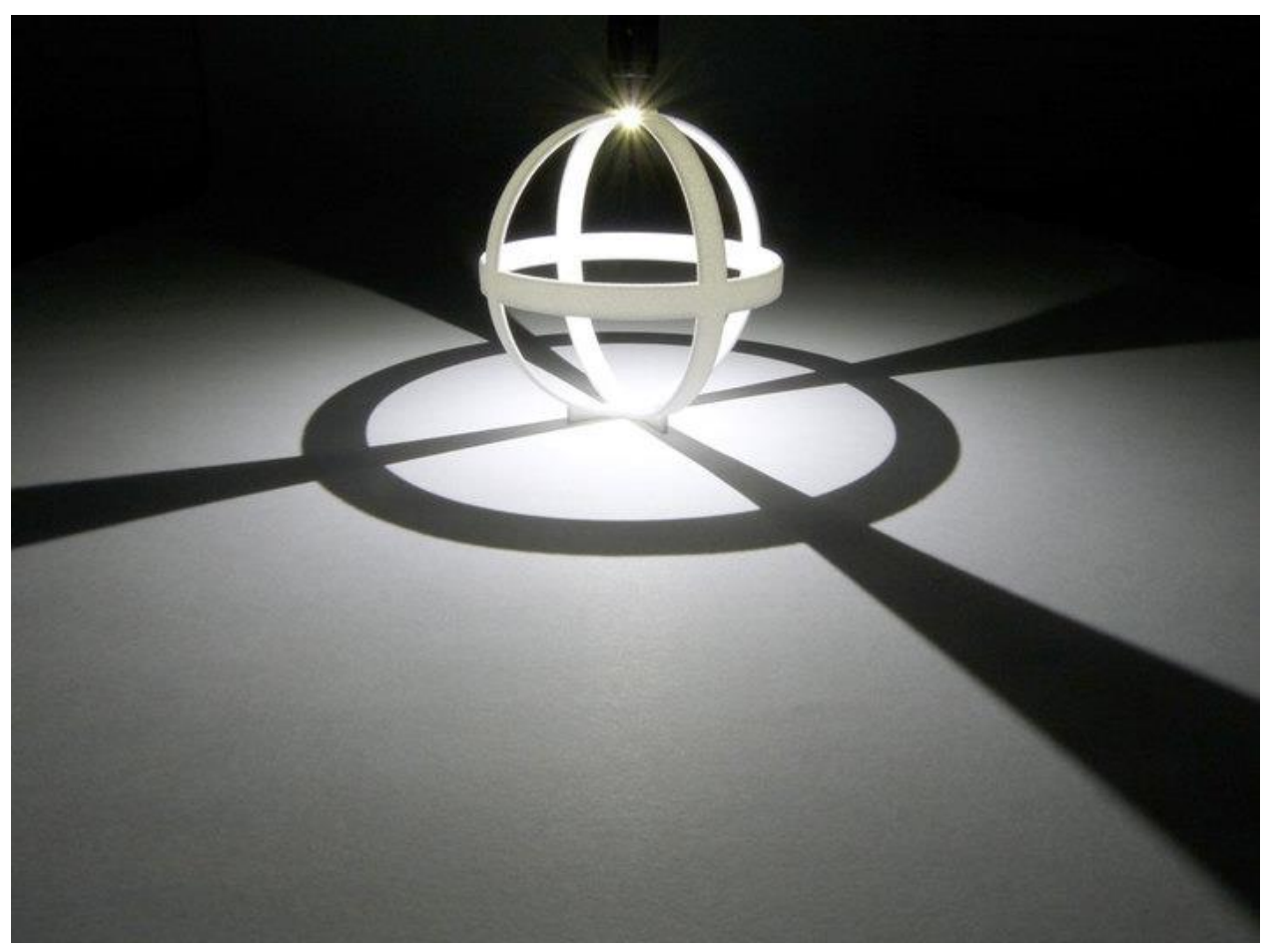

On the real world, a straight line will extend to infinity, and two lines after passing each other will never meet again. But when looking upwards the sphere, they are just images of circles going through the top, repeatedly passing and meeting each other. We see that they not just reunite at infinity, but also reunite their past in the future.

Whenever the sphere spins, everything around us changes. Distant things will move close unexpectedly, and familiar ones will leave us softly. This may seem absurd, but also evident at the same time. Evident, but cannot be grasped, for it comes from a place out of our sight. Cannot explain the unreasonable, nor can explain the obvious, that ambiguity would be frustrating.

Let's gather all the ambiguities altogether, and name it as $x$. With just a simple question, a puzzle piece is flipped. And by perseverance, the symmetry within will emerge. Turns out it's the symmetry. They will run along a circle, imitate the symmetry of the sphere, creating periodic movements, the simplest of which is the pendulum. 


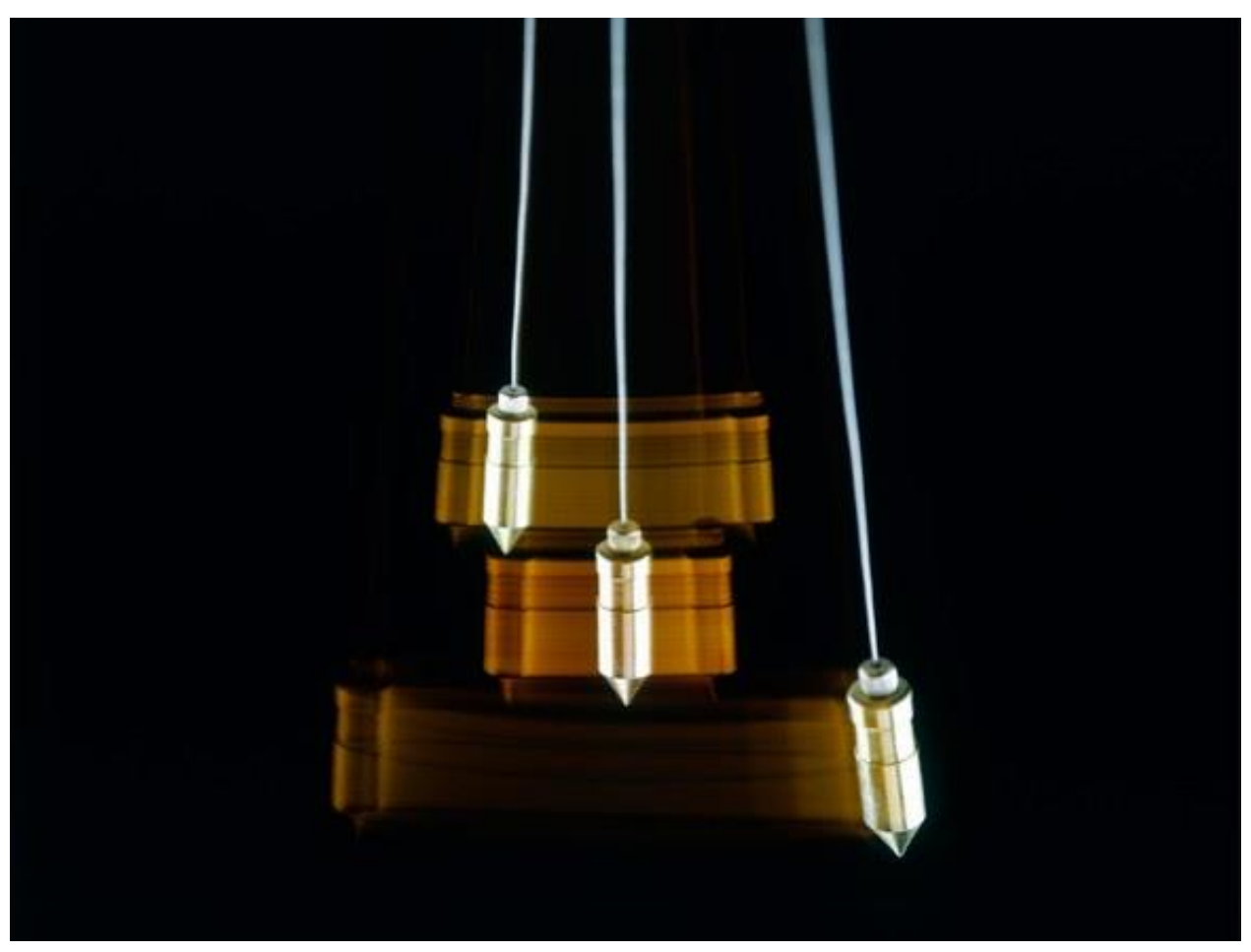

Untold pendulums are imprinted in every single thing, swinging perennially. All things are just combinations of them. Each pendulum has its own rhythm, it will move slowly at two opposite ends, but faster during the middle of the swing. This is why the link between two obvious points is so faded. Sometimes it is so faded that no-one can possibly consider that the two extremes are just the same thing.

It will resonate when acted by its own rhythm. The resonance, even transient, is enough to evince it. The pendulums can also join together to form waves. The waves might be invisible, but can spread out throughout space, recurrent over time, ready to resonate to anything share its rhythm.

The preamble is based on a mathematical idea: the irreducible representation of the Möbius group of the Riemann sphere is homomorphic to the basis of the infinite-dimensional Hilbert space $\mathbb{C}^{\infty}$. As they are complex numbers, they are at the same time the eigenvalues of differential operators, and the harmonic oscillators in physics. Since I self-taught in math, that statement might not be rigorous, but I hope it's not completely false at the same time.

I suppose, when the Buddha enlightened the truth, what he really saw was the Fourier transform. The similarity is striking in Hermann Hesse's Siddhartha. But several thousand years ago, it was very difficult to express this idea, and he had to lay down concepts such as kalpa, nirvana, samsara, impermanence and so on. He himself felt helpless in communicating, as the more he said the wrong it was.

In contrast, when scientists are engrossedly chasing after rigor, they have ignored the feeling of fluidity, flexibility, or transformation. It is the same situation when two people quarrels, one side argues that "rules are rules", and the other says "rules are so strict". No matter what one side says, the other cannot feel satisfied. Both fail to convey the entanglement, the interweave that each of them recognizes it so easily and so difficultly at the same time. What they mean is the movement, but they stuck in concepts. They look at one thing, but they don't speak the same languages. 
In this article, movement, transformation, interweave, entanglement, fluctuation are different names of a same thing; which is used depends on the context.

\section{The fadedness}

Reading Norwegian Forest, I had a feeling that the author is talking about a form of psychological disorder (emotionally unstable personality disorder). And this is how Murakami describe the mood of the character at the beginning of chapter 10:

Thinking back on the year 1969, all that comes to mind for me is a swamp - a deep, sticky bog that feels as if it's going to suck off my shoe each time I take a step. I walk through the mud, exhausted. In front of me, behind me, I can see nothing but the endless darkness of a swamp.

The author describes the exhaustion of the character by comparing it with walking around in swamp. But only readers who actually have experienced that exhaustion, either in the mud or in their real life, can be touched. For those who haven't experienced that, the feeling is not vividly clear. However, has the character used less "I" and focused fully on the stickiness of the mud (since that's the only way an inexperienced reader can imagine), the experience would have been more vivid. An example on turbulent flow:

When a smoke begins to smolder, it first maintains its stability. But with just a little turbulence, the smoke becomes an uncontrollable chaos. Swirling currents will be generated to radiate heat outwardly, which rolls together and causes more and more energy to be lost. And after the energy is completely depleted, it will dissolve into the surroundings and leave not even a single mark behind. 


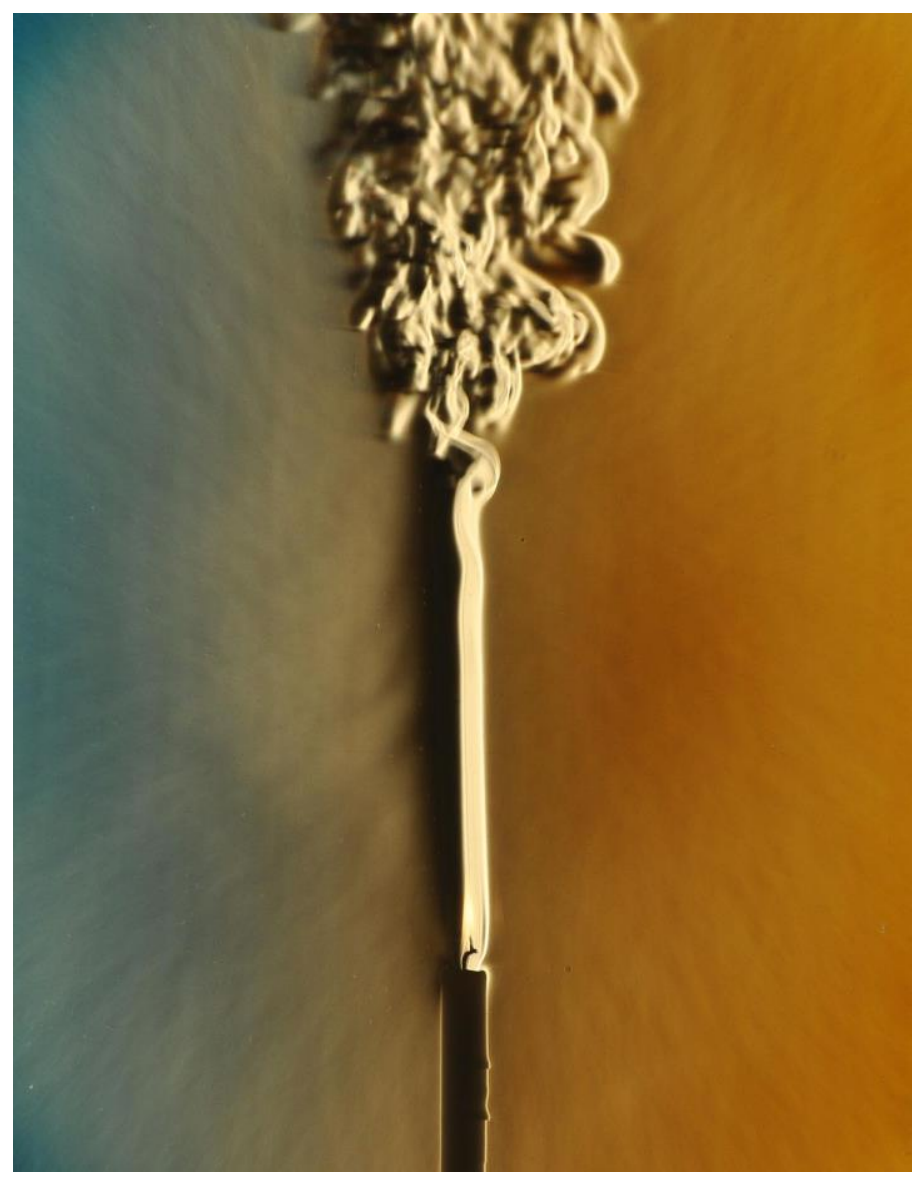

(Notice how the words first, but, with just, little, together, more and more, and after, completely, not even a single although don't serve as key words to understand the concept, they do push them to a higher degree. One by one, the readers experience a chain of small exhaustions. We will return to this shortly.)

When an expert explains a problem to a novice, they tend to give its definition right ahead (like Wikipedia), or compare it with another thing (like "Beethoven is the next Mozart"). But definitions only replace an abstruse word with a sequence of another abstruse words, and comparisons tend to forget the essences of the compared thing ("but Beethoven is not Mozart?") - they are not movements.

Movements, the ways that the objects are transformed fully, completely, thoroughly, entirely, the thing that experts enthusiastically want to give, and the novices most desired thing to receive, is left unnoticed. Until that transformation is achieved, no one is satisfied.

Throughout the entire life of the blind, however curious they are, he cannot use eyes to acquire knowledge. Now if we say that the elephant is an animal with a trunk, then he could never get it. And when he investigates the elephant by himself, he couldn't comprehend an animal that sometimes like a snake, sometimes like a tree. But perhaps showing the transformation of the elephant will enlighten him:

When a thing that sometimes like a snake, sometimes like a tree, then we know that it's an elephant. 
He may still wonder why the elephant is so miraculous as that, but he will no longer find it vague, even when he just knows only two features of the elephant. For him, "like a snake" and "like a tree" are already clear and nothing's special, but the link between that two clear states are extremely faded, and that's where he gets stuck. It's that fadedness connects all the opposites points into one. The entire meaning of the "elephant" concept lies in there. Once understood, then we can add more information to better paint the picture. These can be whatever additional details: the tusk, its tail, the weight, its relationship with human cultures, etc.

Named "faded", meaning it never appears clear. Using more accurate words or adding more detail only give us a momentary clearness at that time, but the feeling is fleeting. It's like picturing the pendulum at the middle of the swing, the result is just another clear image; the fadedness of the whole movement has disappeared.

Note that I'm not saying that seeking for accurate words is useless. As how Mark Twain said:

The difference between the almost right word and the right word is really a large matter-it's the difference between the lightning bug and the lightning.

"The almost right word", "the right word", "the lightning bug" and "the lightning" are all the right words/phrases for this idea. But the meaning doesn't lie within those words, it lies on the comparisons between:

- the lightning bug and the lightning

- the almost right word and the right word

- the almost right word and the right word and the lightning bug and the lightning

Another good example is a couplet from Poincaré and an unknown poet:

Mathematics is the art of giving the same name to different things

Poetry is the art of giving different names to the same thing

Or in the turbulence prose, the linking words provide the context for the readers. One can temp to say that the meanings lie in the prepositions, conjunctions, punctuation marks, grammar, typography or context, and in this case it's not wrong. But it makes no sense to say the all the meaning is in them; the fadedness has slipped back to the right words. This is essentially the Taoist philosophy: the true Tao cannot be captured by language. This philosophy permeates East Asian cultures, from hyponymous juxtapositions in language to antithetical couplets in culture. The same goes for translation, in which translators have to forget what they just read in source texts to have natural translations.

Perhaps all math books, even when the authors are so actively aware of the big picture, falls into the trap of clearness ${ }^{123}$. Let's take Kreyszig's Introductory Functional Analysis and Wu-Ki Tung's Group Theory in Physics for instance:

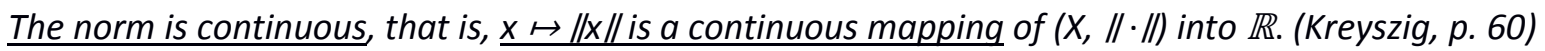

A representation $U(G)$ on $V$ is irreducible if there is no non-trivial invariant subspace $V$ with respect to $U(G)$. (Wu-Ki Tung, p. 33)

\footnotetext{
${ }^{1}$ Commonly known as the curse of knowledge

${ }^{2}$ I am of course can always, and will always avoid to be an exception

${ }^{3}$ The only exception I know is Needham's Visual Complex Analysis.
} 
Example 1 has redundant words and symbols. Example 2 is a neat reference for experts, but for a learner it compresses too much new terms into one sentence, not to mention that they are all in negative form. I would rewrite them as:

Norm is a continuous mapping into $\mathbb{R}$.

If a representation on a space is reduced to the point that only that space and $\{0\}$ are its only two subspaces that can hold their vectors inside them, then the representation is irreducible.

My experience is to avoid using let and to be, only use popular symbols (e.g. $\mathbb{C} \infty, \operatorname{PSL}(2, \mathbb{R}))$, move the term to define at the end of sentence or convert it to adjective or verb, and directly use the definitions of the terms build up it. Also, the logic is just a movement of the clearnesses, so the relations should be a fadedness, not clearness. For example:

\begin{tabular}{|l|l|}
\hline Relation & Translation \\
\hline$=$ & must be equaled to; can be seen as \\
\hline $\begin{array}{l}\text { Homomorphism } \\
\left(G \text { is homomorphism with } G^{\prime}\right)\end{array}$ & put G into G' \\
\hline$\Leftrightarrow$ & has the same meaning with \\
\hline $\begin{array}{l}\text { If } X \text { happens then (and only } \\
\text { then) } Y \text { happens }\end{array}$ & $\begin{array}{l}Y \text { always exists if } X \text { exists; } Y \text { will never appear unless there is } X \text {; once } Y \\
\text { doesn't exists then } X \text { doesn't happens in the first place }\end{array}$ \\
\hline
\end{tabular}

The point is to reach the mindset that everything is so obvious that there is nothing needs to be defined at all. By keeping the definitions of the terms to be embedded inside the flow, the dynamic of these terms will emerge. This will inevitably invoke wrongness or ambiguities in the experts' eyes, but I hope this is acceptable, in the sense that whatever they point to and say "this is wrong/unclear" they will realize that it is locally and can be refined easily. Likewise, when choosing books I usually imagine the book is a painting, yet I forget to bring my eyeglass. If every time I close my eyes and reopen them I see a new painting, yet I still don't feel vague with it, then that book is worth reading.

Question:

- Social sciences. How does semantics, pragmatics and discourse analysis in linguistics view this? How does critical theory view this?

\section{Physical model}

My theory on semantic information is based on an observation that objects only becomes visually clear when their velocities are approximately zero. What physical model can describe this?

Strictly speaking, it's the relative velocity should be zero: 


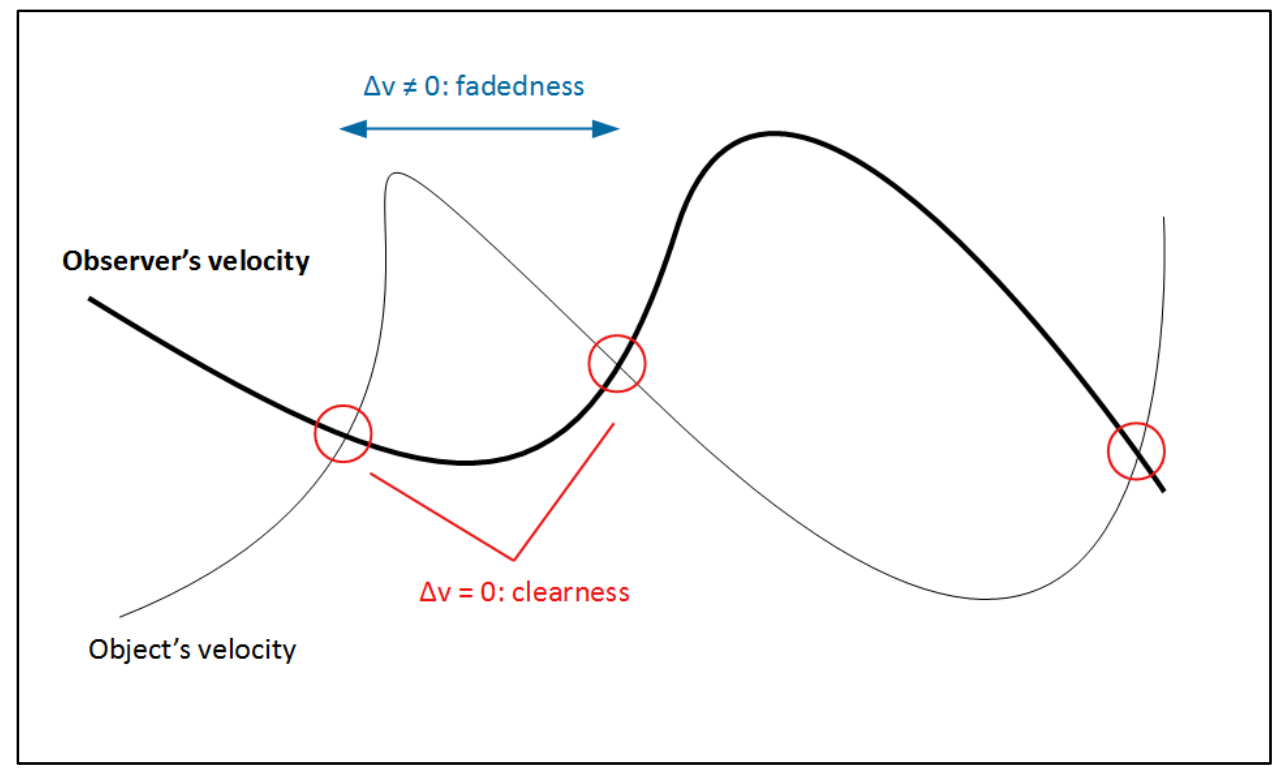

Or equivalently:

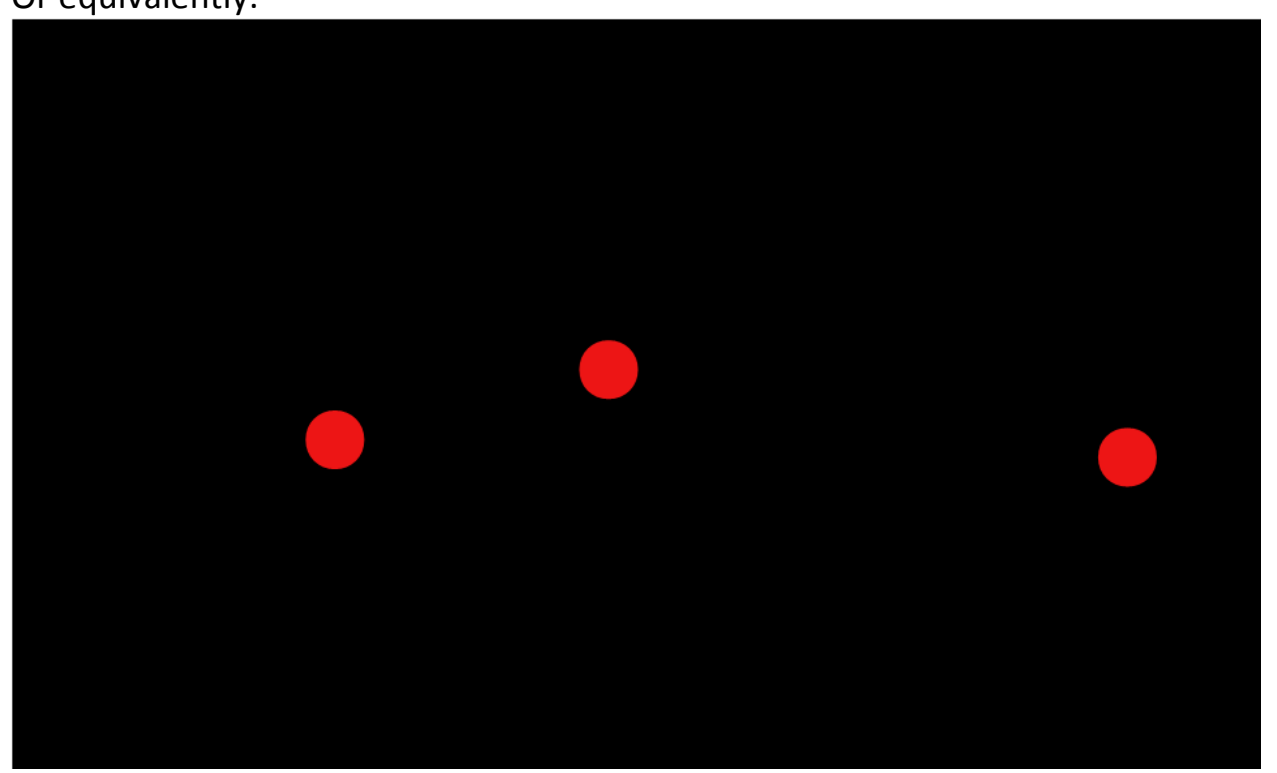

Seeing only those dots is not enough to postulate anything, however to see other dots the observer needs to change their velocity, therefore losing current clear dots. While velocity is a central concept in both dynamical systems theory and special relativity, as far as I know they have only been developed with an implicit assumption that objects can always be seen in any velocity. I think this assumption closely relates with the assumption that position and velocity can be measured at the same time.

The clearness is the solution of $\mathrm{dx}_{\text {observer }} / \mathrm{dt}=\mathrm{dx} \mathrm{x}_{\text {object }} / \mathrm{dt}$.

\section{How clear?}

In the case which $v_{\text {observer }}=0$ and the object is a single pendulum, the clearnesses is also the extremums in amplitude, so we can use amplitude graph instead. This will not only capture the contrast by the difference between maximums and minimums, but also compare the significance of movements. For example, one can assume that the difference between the almost right word and the right word is harder to notice than the difference between the lightning bug and the lightning. So: 


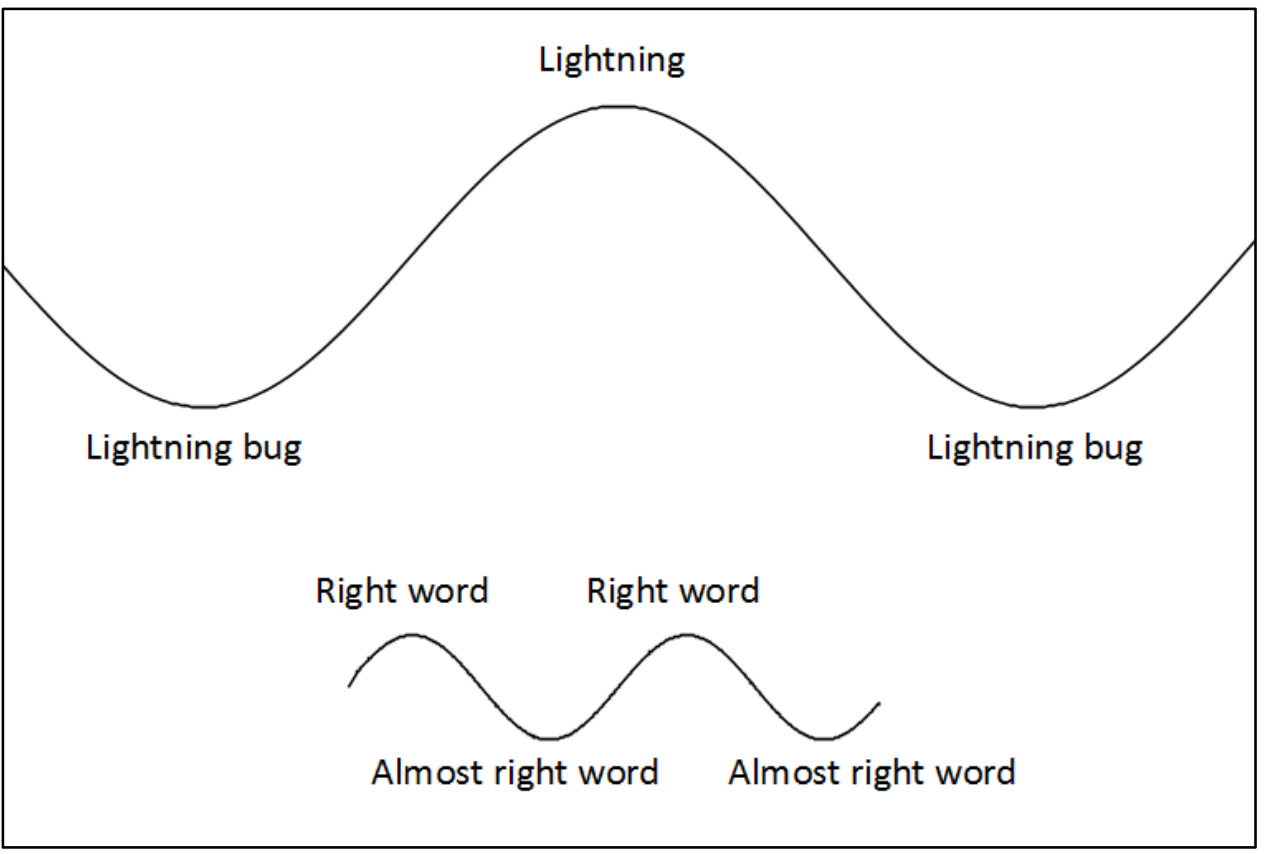

If the object has two oscillations, then there are moments that the lower frequency oscillator is faded, while the higher one is clear, and the object is in the half faded half clear state. During the movement, if the higher one has already change to another clear position, but the lower one is still faded, then it's just a temporary fluctuation. It's only wholly clear when all components reach maximums or minimums at the same time.

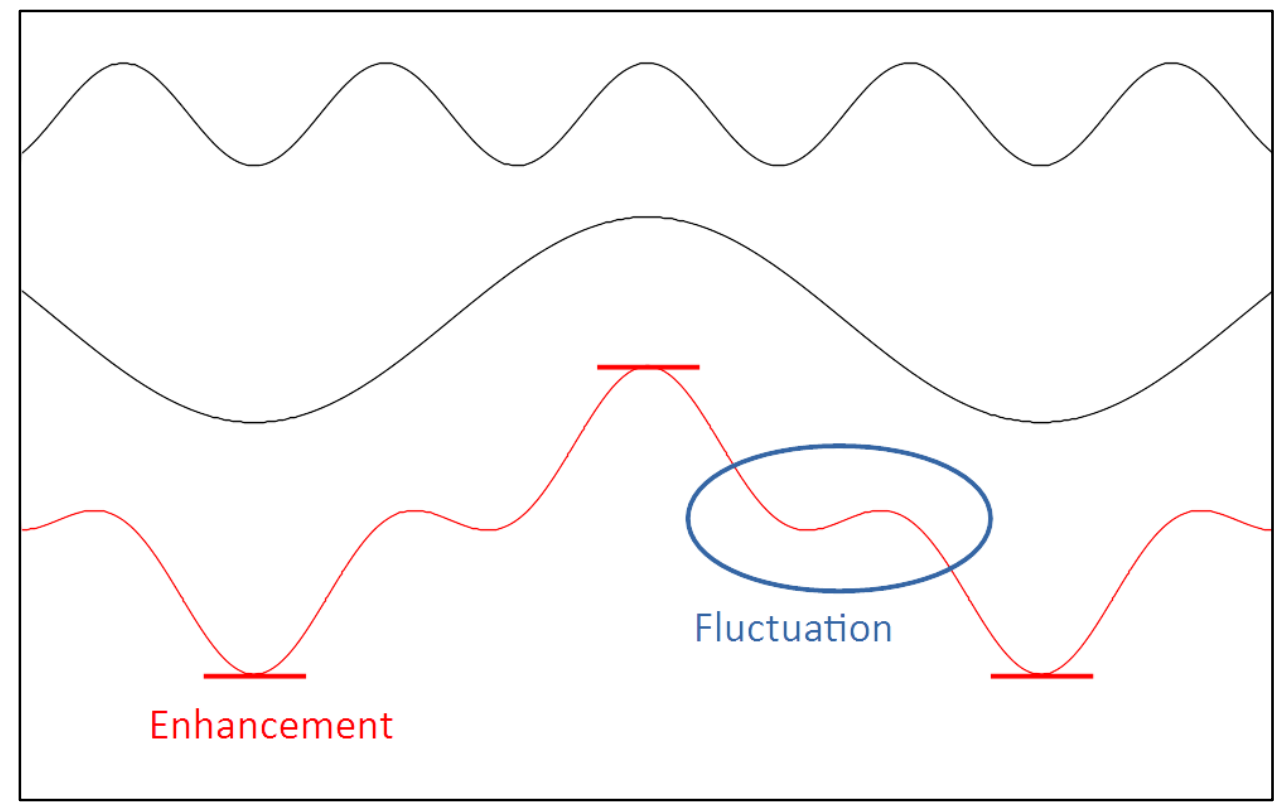

Extremums happen when all components align in the same phase, so the movement is enhanced to escape the fluctuation: 


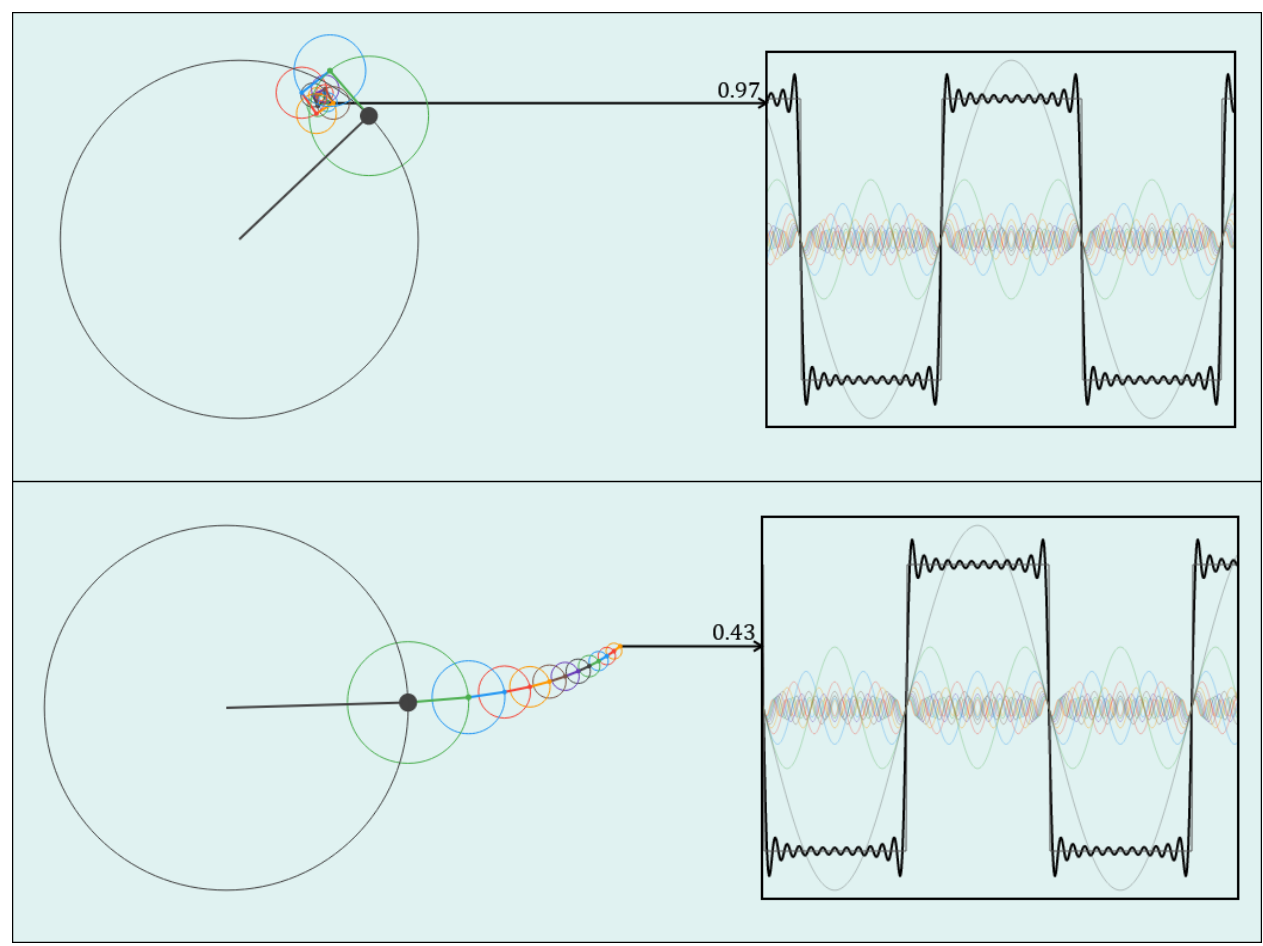

Note that the square wave and its jumps are just for demonstration, in reality most waves don't behave like this.

Question:

- Harmonic analysis:

$\circ \quad$ Frequency. How to identify the frequencies? How about the Doppler effect?

- Superposition. How about combining two vibrations perpendicularly? How would the fadedness behave in higher dimensions?

$\circ$ Dynamical. What happens if during fluctuation, the velocity passes the horizontal axis?

- Complex systems theory:

- Emergence. Is the alignment of the oscillators what we call emergence? Are optimization, evolution, specialization, computation just different names of the changes of waves when new oscillators are added?

- Information. Is the amplitude graph the spatial distribution and temporal dynamics of living systems like ant colonies or immune system? Can Fourier analysis be applied in hormone concentrations, or ecological populations?

- Purpose. Since oscillators are amplified only when their frequencies are matched, does this explain why there are things that are more important to others, and the purpose of the system is to make as many resonances as possible?

\section{How faded?}

When choosing books I usually imagine the book is a painting, yet I forget to bring my eyeglass. If every time I close my eyes and reopen them I see a new painting, yet I still don't feel vague with it, then that book is worth reading. 


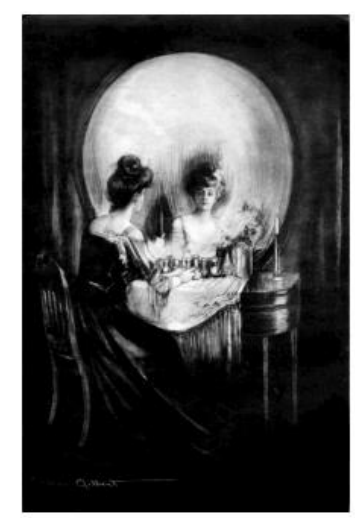

(a)

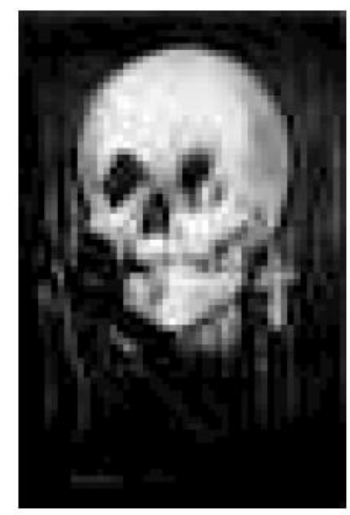

(b)

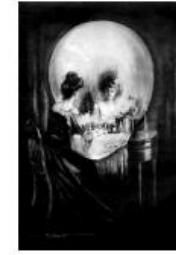

(c)

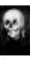

(d)

Figure 7.2 Interplay of scale and resolution. (a) The original, high-resolution version. (b) A blurred, lower-resolution version; the resolution is lower and thus our perception of the image has changed. (c) A scaled version of half size in each dimension; the resolution is unchanged and thus our perception of the image remains unchanged as long as our visual acuity is high enough. (d) A version scaled further, to the point that our perception of the image changes (we see only the skull) since our visual acuity is not high enough to capture details.

This is an excerpt from the chapter Localization and uncertainty of the book Foundations of Signal Processing. It suggests that fadedness has also been well researched in the field of signal processing with the Fourier transform, hence this can be the bridge to Shannon's information. The point is not about how a bunch of decentralized interactions of large number of simple neurons recognize a bunch of pixels as the beauty of the woman's face reflected in the mirror, but about how those pixels and neurons "move".

Questions:

- Information. Is fadedness a physical phenomenon, not just a cognitive phenomenon? This would mean that even when I'm not aware of your existence, but as long as a part of me has the same velocity of a part of you, then there is an information about you that I have. And we can only "understand" each other if we coincidently walk the same velocity for a suffice amount of time.

- Cognitive science. What would be the movement inside our minds and the skull image above? The velocity of this movement should be in $\mathrm{m} / \mathrm{s}$ unit. Is the fadedness what we called "subconsciousness"?

\section{- Relativity theory - quantum mechanics:}

- If fadedness is a different name of relative velocity, does it mean special relativity effects can be observed in human scale?

- If fadedness is a different name of uncertainty, does it mean quantum effects can be observed in human scale?

- If uncertainty and relative velocity are different names of fadedness, does this mean quantum effects are contained in special relativity and vice versa? 


\section{L: amplitude of lightning bug - lightning oscillator W: amplitude of almost right word - right word oscillator}

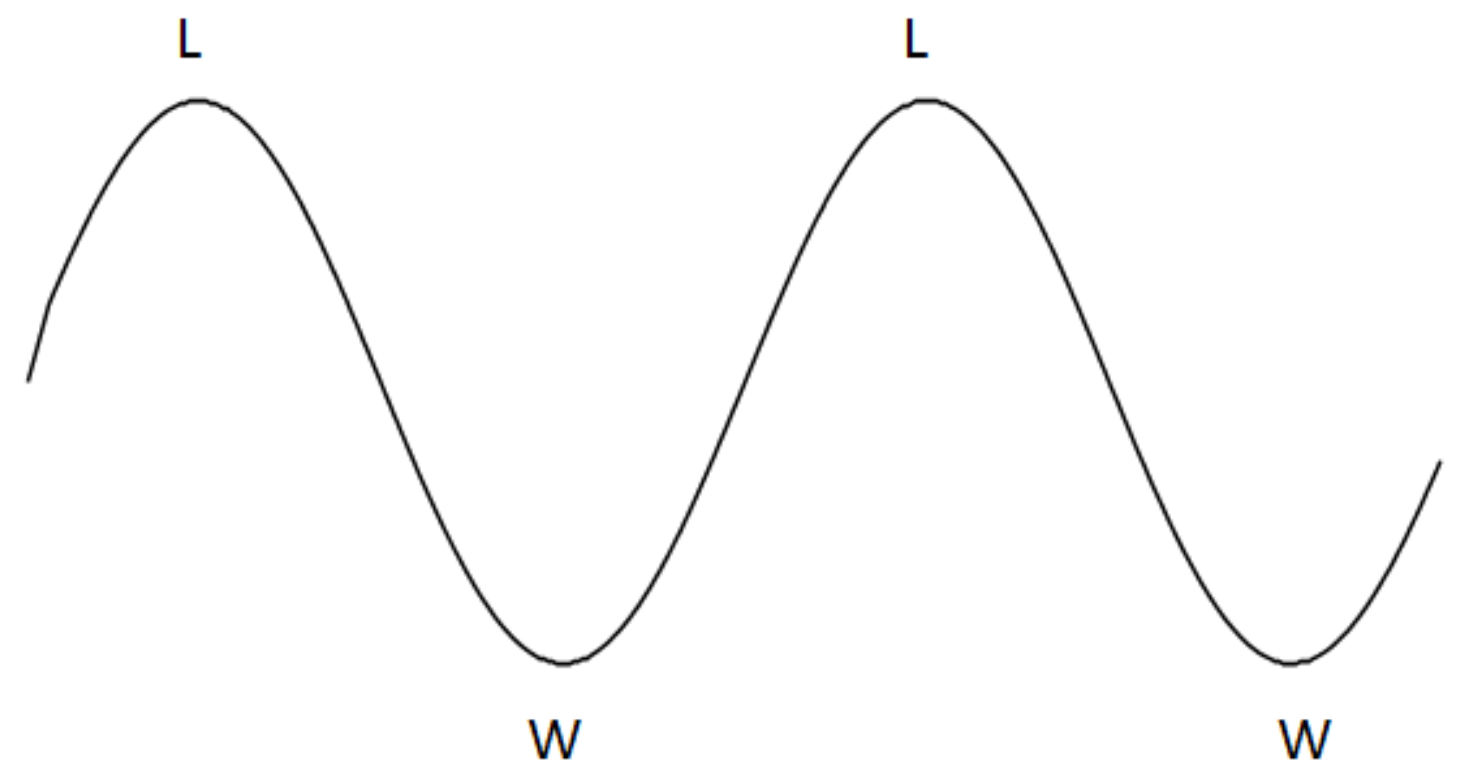

$\mathrm{L}$ and $\mathrm{W}$ are movements themselves, but in this oscillation they are just clear points. Giving scaled down version of the skull image, no one can imagine the eyes are actually the woman's face and her reflection. For those who has experienced helplessness in life, the Murakami's paragraph is touching. In the previous section I has hypothesized that the clearnesses contains no useful meaning; meaning is only contained in the fadednesses in the movements. In this section I will develop a second hypothesis: the whole movement can be perceived as a clear point, and a clear point contains a whole movement within if it is studied long enough.

Life or death, right or wrong, love or hate, they are all perceived as clear as black and white. Poets' mot justes or scientists' terms, or convincing discourses like "electrons spin around the nucleus like the solar system" or "all men are created equal" all share the same characteristic: they are all born to solve a paradox created by two clear things, but then they become another clear thing, equal to the old clear things contribute to it. Looking at any industry and one can see countless innovations has been invented to solve that much preoccupations in their times, but when looking back they are just arrangements of oscillators. Explaining a joke kills it. 50 years later, that elephant is just another passing animal in the mind of the old blind man, no longer a miracle anymore.

Why can those movements become clearness? Why can a whole trajectory of one pendulum be equivalent to one extreme of another pendulum? Does this relate to coupled oscillators, where one stand still when the other moves? Why can we can describe a pendulum as a complex number, even 
though it's not truly an oscillator from standard model? Is this a kind of large-scale oscillation? Some possible explanations I can think of:

1. This is a kind of self-replication (fractal)

2. It depends on the projective plane (projective geometry)

3. Each oscillator has a "dual/adjoint" oscillator (harmonic analysis)

I don't know much about fractal, unfortunately.

\section{Projective geometry}
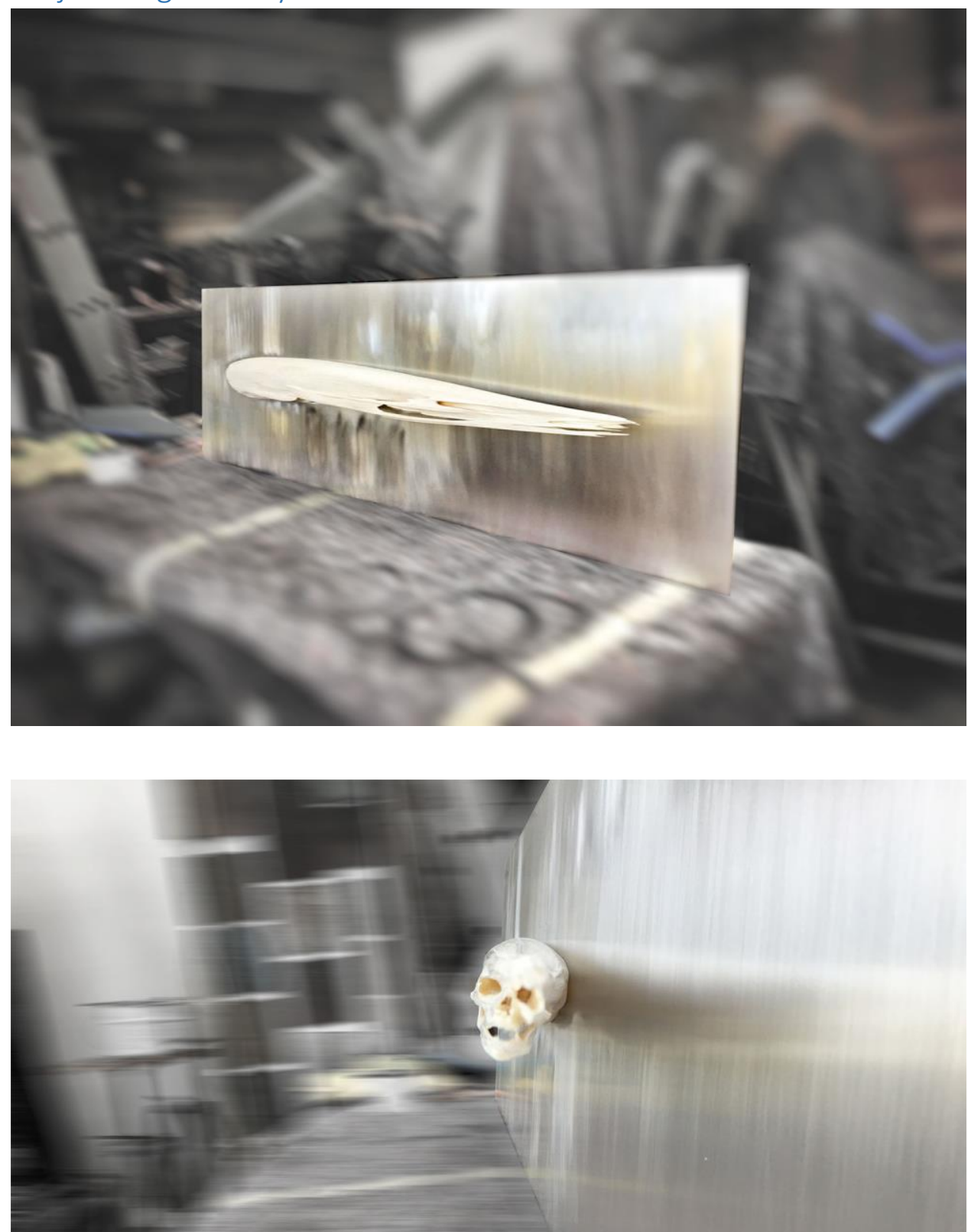

(Ugh, not the skull again. But this is the only example I can find that can illustrate the movements in any perspectives. Other artworks only focus on distortion.) 
In simple words, projective geometry is about how shapes are distorted when perspective changes. So a spreading movement can be shrunk to a dot and vice versa. It is striking because in projective geometry the role of points and lines can be interchanged in definitions and theorems (principle of duality). Projective geometry is central in computer vision, and... relativity theory. In art, that style is called anamorphosis.

Note that the skull is not the meaning of the sculpture; all perspectives are created equal. The reason why the latter image is more equal than the former is because it matches with our movement.

Questions:

- Philosophy: is this a kind of deconstructionism, as there is no such a thing as the true meaning of object? However, logocentricism is possible, as long as the observer and the object have the same velocity. However again, since both are in a constant state of flux, that moment is fleeting, and the meaning is deferred. Saying logocentricism is possible, does not mean it's easy to get. In other words, if deconstructionism and logocentricism are binary opposition, then these concepts are deferred.

- Mathematical psychology: how does this relate to Louis Narens' work on meaningfulness on measurement theory? Erlanger program and axiom of choice are used.

- Complex systems theory:

- Randomness. Is randomness a consequence of the rotation of the Riemann sphere? Randomness is the source of diversity; without it one cannot explore what to explore.

- Complexity. If there is no layer deeper than others at all, does defining or measuring "complexity" become theoretically impractical, though practically useful? In one perspective, an Al has more movements than a cat, but in another one, the cat has more movements than the Al.

\section{Harmonic analysis}

Duality also happens in harmonic analysis: Pontryagin duality. Would the fact that each oscillator is the basis of a 1-dimensional space, and there is a dual space for any vector space, explain the existence of dual oscillator? Is our world a representation of a locally compact group? Is the double dual oscillator a new or an old one? How does this duality relate to projective geometry?

If this duality can be seen in language, then would it mean that mathematical ideas are just oscillators, despite the fact that they are more and more abstract and far away from the classical Fourier transform? One supported evidence is that harmonic analysis permeates mathematics. Even when Higgs boson is found, let alone when the tachyon will ever be found, isn't that the LHC is only more advanced than a hoe by the number of oscillators? And we know that the number of oscillator is infinity.

If a mathematical proof is really no different than a music sheet, then this can explain why Einstein saw his life in terms of music, why mastered mathematicians can rely on their intuition to guide them, like how tempo keeps you on track. Local errors will automatically cancel out another local error to yield correct results. If sophisticated knowledge can really be different than oscillators, then there is no need to use reputation to filter out bad ideas or to reorganize ideas after a period of research. There even won't be a thing called "idea" at the first place.

Question: 
- Quantum field theory. How does quantum field theory connect harmonic analysis with projective geometry? Is there a connection between two dualities? Do resonances or enhancements change perspectives? $U(1)$, the gauge group of quantum electrodynamics, and $\mathbb{R} U\{\infty\}$, the projectively extended real line, are actually just different names of the circle.

- Psychology. From bearing stomach ulcers for persuading a dream, to having uncontrollable rages as a consequence of an unfortunate childhood, all are to see something clear inside. Are they the results of wave fluctuations? If we are just a combination of oscillators, then does this explain why everyone has different hobbies, beliefs, balance points, and each person has to find their own way, since nobody can know all the oscillator in other people? On that way new oscillators will join us, and old oscillators are more and more resonated.

I think music is the best way to answer this. The answer is blowin' in the wind.

\section{Summary}

- Clearness is the result of zero velocity or enhancement

- Fadedness connects clear points to make a meaning

- A clear point can become a movement, and vice versa

\section{Some important questions}

- Emergence. Is the alignment of the oscillators what we call emergence? Are optimization, evolution, specialization, or computation just different names of the changes of waves when new oscillators are added?

- Cognitive science. What would be the movement inside our minds and the skull image above? The velocity of this movement should be in $\mathrm{m} / \mathrm{s}$ unit. Is the fadedness what we called "subconsciousness"?

- Philosophy: is this a kind of deconstructionism, as there is no such a thing as the true meaning of object? However, logocentricism is possible, as long as the observer and the object have the same velocity. However again, since both are in a constant state of flux, that moment is fleeting, and the meaning is deferred. Saying logocentricism is possible, does not mean it's easy to get. In other words, if deconstructionism and logocentricism are binary opposition, then these concepts are deferred.

- Randomness. Is randomness a consequence of the rotation of the Riemann sphere? Randomness is the source of diversity; without it one cannot explore what to explore.

- Complexity. If there is no layer deeper than others at all, does defining or measuring "complexity" become theoretically impractical, though practically useful? In a perspective, an Al has more movements than a cat, but in another one, the cat has more movements than the Al.

- Quantum field theory. How does quantum field theory connect harmonic analysis with projective geometry? Is there a connection between two dualities? Do resonances or enhancements change perspectives? $U(1)$, the gauge group of quantum electrodynamics, and $\mathbb{R} U\{\infty\}$, the projectively extended real line, are actually just different names of the circle. 
Diagram

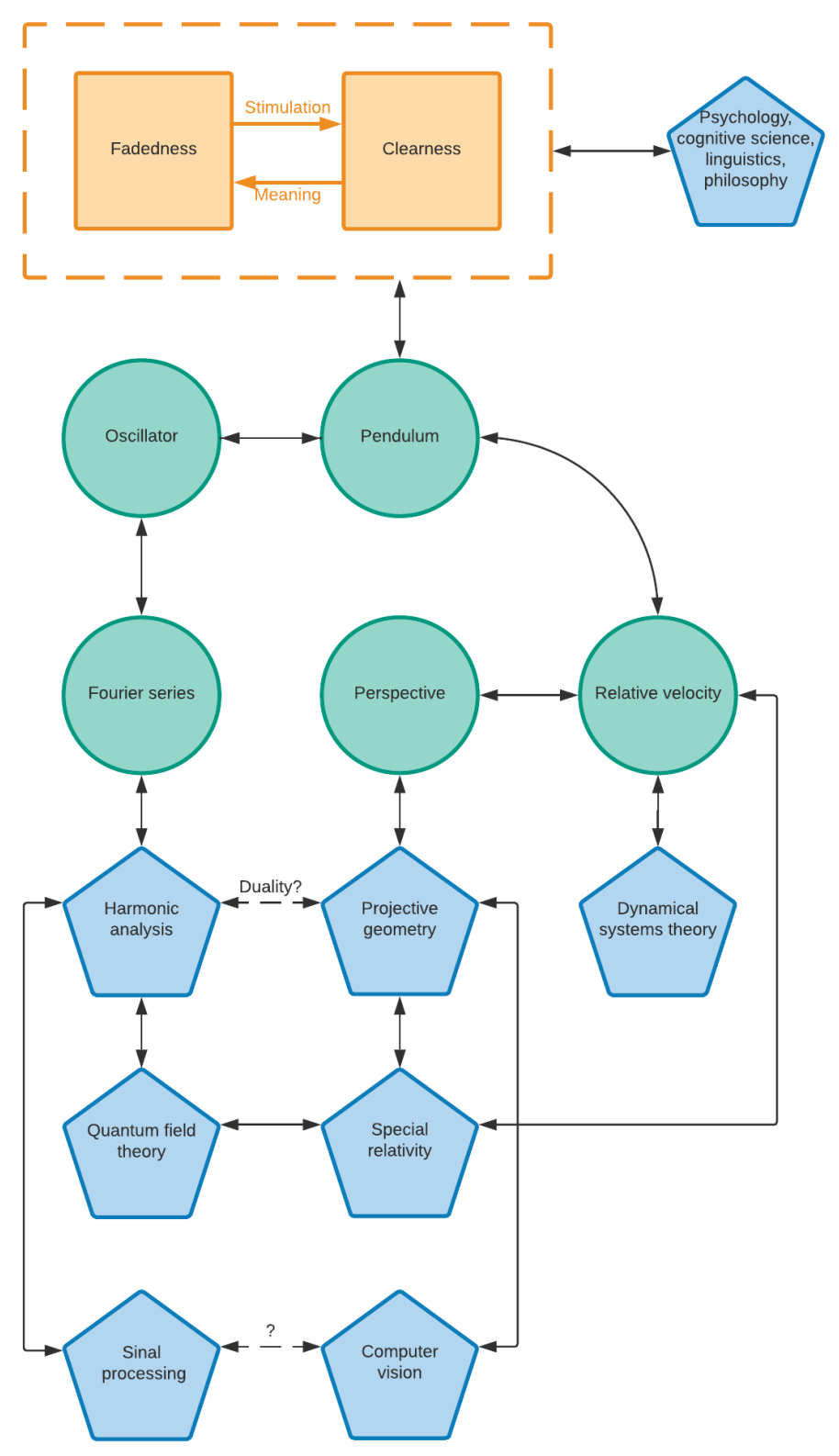




\section{Appendix}

\section{Last chapter of Hermann Hesse' Siddhartha}

"Siddhartha," he said, "we have become old men. It is unlikely that either of us will ever see the other again in this shape. I can see, beloved friend, that you have found peace. I confess that I myself have not done so. Grant me just one word more, O Revered One; give me something that I can grasp, that I can comprehend! Give me something to take with me when we part. My path is often difficult, Siddhartha, often dark."

Siddhartha remained silent and continued to gaze at him with the same still smile. Govinda stared into his face with fear, with longing. Suffering and eternal searching stood written in his gaze, eternal notfinding.

Siddhartha saw this and smiled.

"Bend down to me," he whispered softly in Govinda's ear. "Bend down here to me! Yes, like that, closer! Even closer! Kiss me on the forehead, Govinda!"

When Govinda, perplexed and yet drawn by great love and foreboding, obeyed his words, bent down close to him, and touched his forehead with his lips, something wondrous happened to him. While his thoughts were still lingering over Siddhartha's odd words, while he was still fruitlessly and reluctantly attempting to think away time, to imagine Nirvana and Sansara as one, while a certain contempt for his friend's words was even then battling inside him with tremendous love and reverence, this happened:

He no longer saw the face of his friend Siddhartha; instead he saw other faces, many of them, a long series, a flowing river of faces, by the hundreds, by the thousands, all of them coming and fading away, and yet all of them appearing to be there at once, all of them constantly changing, being renewed, and all of them at the same time Siddhartha. He saw the face of a fish, a carp, its mouth wrenched open in infinite pain, a dying fish with dying eyes - he saw the face of a newborn child, red and full of wrinkles, all twisted up to cry-he saw the face of a murderer, saw him stick a knife into a person's body, and saw, at the same instant, this criminal kneeling down in chains and having his head chopped off by an executioner with one stroke of the sword-he saw the bodies of men and women naked in the positions and struggles of furious love-he saw corpses laid out, still, cold, empty-he saw the heads of animals: wild boars, crocodiles, elephants, bulls, birds-he saw gods, saw Krishna, saw Agni-he saw all these figures and faces in their thousandfold interrelations, each helping the others, loving them, hating them, destroying them, giving birth to them anew; each one was a wanting-to-die, a passionately painful confession of transitoriness, and yet none of them died; each of them was only transformed, constantly born anew, constantly being given a new face, without time having passed between one face and the next-and all these figure and faces rested, flowed, engendered one another, floated off and streamed into and through one another, and constantly stretched over all of them was something thin, an insubstantial but nonetheless existing thing like thin glass or ice, like a transparent skin, a bowl or shape or mask made of water, and this mask was smiling, and this mask was Siddhartha's smiling face, which he, Govinda, at just this moment was touching with his lips. And Govinda saw that this smiling of the mask, this smile of Oneness over all the flowing figure, this smile of simultaneousness over the thousand births and deaths, this smile of Siddhartha was precisely the same, was precisely the same still, delicate, impenetrable, perhaps kind, perhaps mocking, wise, thousandfold smile of Gautama, the Buddha, as he himself had seen it a hundred times with awe. This, Govinda knew, is how the Perfect Ones smiled.

No longer knowing whether time existed, whether this looking had lasted a second or a hundred years, no longer knowing whether there was a Siddhartha, whether a Gautama, whether a Self, an I and You, wounded in his innermost core as if by a divine arrow whose wound tastes sweet, entranced and 
bewildered in his innermost core, Govinda remained standing there a short while longer, bending over Siddhartha's still face that he had just kissed, that had just been the site of all shapes, all Becoming, all Being. This countenance appeared unchanged once the depths of the thousandfold immensity had closed again beneath its surface; he was silently smiling, smiling quietly and gently, very kindly perhaps, perhaps mockingly, precisely as he had smiled, the Sublime One.

Deeply Govinda bowed, tears of which he knew nothing coursed down his old face, and like a fire the feeling of the most ardent love, the most humble reverence was burning in his heart. Deeply he bowed, bowed to the very earth, before the one sitting there motionless, whose smile reminded him of everything he had ever loved in all his life, everything that had ever, in all his life, been dear to him and holy.

\section{A quote from Hardy}

I have myself always thought of a mathematician as in the first instance an observer, a man who gazes at a distant range of mountains and notes down his observations. His object is simply to distinguish clearly and notify to others as many different peaks as he can. [...] But when he sees a peak he believes that it is there simply because he sees it. If he wishes someone else to see it, he points to it, either directly or through the chain of summits which led him to recognize it himself. When his pupil also sees it, the research, the argument, the proof is finished.

The analogy is a rough one, but I am sure that it is not altogether misleading. If we were to push it to its extreme we should be led to a rather paradoxical conclusion; that there is, strictly, no such thing as mathematical proof; that we can, in the last analysis, do nothing but point; that proofs are what Littlewood and I call gas, rhetorical flourishes designed to affect psychology, pictures on the board in the lecture, devices to stimulate the imagination of pupils. This is plainly not the whole truth, but there is a good deal in it.

\section{A short prose}

You are now having a joyful delightful story in this very minute, and would like to see it last forever without having to worry about losing it one day? Ask questions. Many many questions. The answers to your rumination will always be within your reach, because you are not alone, because you are not helpless. A faint full of warmth will gently weave into every corner of your soul, and in every living moment, telling what you have been longing for will come with you in the most unexpected way. Better still, ask unimaginable questions. At that time, for whatever happens to you, a smile can always radiate on your face, since in your hands there are countless paths to continue the journey. 


\section{Bibliography}

\section{Mathematics}

Projective geometry

Arnold, Douglas, and Jonathan Rogness. Mobius Transform Revealed

Hartley, R., and A. Zisserman. Multiple View Geometry in Computer Vision. Cambridge University Press, 2004

Henry Segerman. Octahedron (Stereographic Projection)

Jonty Hurwitz. Tribute To Holbein.

Needham, T. Visual Complex Analysis. Clarendon Press, 1998.

Stillwell, J. The Four Pillars of Geometry. Springer New York, 2005.

Narens, L., Theories of Meaningfulness, Scientific Psychology Series (Lawrence Erlbaum Associates, 2002)

Dynamical systems theory

Mike Dunning. Pendulum.

Mitchell, M. Complexity: A Guided Tour. Oxford University Press, 2009.

Settles, Gary. Laminar-Turbulent Transition. 2009

Strogatz, S. H. Nonlinear Dynamics and Chaos: With Applications to Physics, Biology, Chemistry and Engineering. Westview, 2000.

\section{Harmonic analysis}

3Blue1Brown. The More General Uncertainty Principle, beyond Quantum. 2018.

Ben Grawi. Understanding Fourier Approximations \& Oscillators. 2016, http://bgrawi.com/FourierVisualizations/.

Dym, H., and H. P. McKean. Fourier Series and Integrals. Academic Press, 1972.

French, A. P. Vibrations and Waves. Norton, 1971.

Kreyszig, E. Introductory Functional Analysis with Applications. Wiley, 1989.

Ooker. "What Does S ${ }^{1}$ Do in Many Branches of Math?" Mathematics Stack Exchange.

Ramond, P. Group Theory: A Physicist's Survey. Cambridge University Press, 2010.

Schwichtenberg, J. Physics from Symmetry. Springer International Publishing, 2015.

Tao, Terence. Fourier Transform.

Tung, W. K. Group Theory in Physics. World Scientific, 1985.

Vetterli, M., et al. Foundations of Signal Processing. Cambridge University Press, 2014.

\section{Cognitive science}

\section{Emotionally unstable personality disorder}

Kreisman, J. J., and H. Straus. I Hate You, Don't Leave Me: Understanding the Borderline Personality. Avon Books, 1991. 
Mason, P., and R. Kreger. Stop Walking on Eggshells: Taking Your Life Back When Someone You Care About Has Borderline Personality Disorder. New Harbinger Publications, 2010.

Sasson, Ofir. Borderline Personality Disorder (2017). https://youtu.be/BFop1UxiDdY.

Veritasium, Learned Helplessness. https://www.youtube.com/watch?v=YMPzDiraNnA.

Language acquisition

Yule, G. The Study of Language. Cambridge University Press, 2014.

Hirsh, David, and Paul Nation. "What Vocabulary Size Is Needed to Read Unsimplified Texts for Pleasure?" Reading in a Foreign Language, vol. 8, no. 2, 1992, pp. 689-96.

Laozi. Daodejing.

Mattos, Maria Teresa Segarra Costaguta. "An Analysis on the Validity of the Lexicon Required by GRE ${ }^{\circledR}$ Test Takers." BELT - Brazilian English Language Teaching Journal, vol. 3, no. 1, Oct. 2012.

Yip, P. The Chinese Lexicon: A Comprehensive Survey. Routledge, 2007.

Wyner, G. Fluent Forever: How to Learn Any Language Fast and Never Forget It.

Potter/TenSpeed/Harmony, 2014.

Liturature - philosophy - metamathematics

Jullien, F. The Propensity of Things: Toward a History of Efficacy in China. Translated by J. Lloyd, Zone Books, 1999.

Keyser, Cassius Jackson. Mathematics as a Culture Clue: And Other Essays. Scripta Mathematics, Yeshiva Univ., 1947.

Laurel. "Was There a Person Made the Quote about Poetry That Poincaré Responded To?" Skeptics Stack Exchange.

Morris, D. The Human Zoo. Random House, 2009.

Tao, Terence. "What Is Good Mathematics?" ArXiv:Math/0702396, Feb. 2007. arXiv.org.

Tao, Terence. "There's More to Mathematics than Rigour and Proofs." What's New, 6 May 2007, https://terrytao.wordpress.com/career-advice/theres-more-to-mathematics-than-rigour-and-proofs/.

Appignanesi, R., C. Garratt, Z. Sardar, and P. Curry, Postmodernism for Beginners, Beginners Series (Totem Books, 1995) 